\title{
Homocysteine and ischaemic stroke in men: the Caerphilly study
}

\author{
U B Fallon, P Elwood, Y Ben-Shlomo, J B Ubbink, R Greenwood, G Davey Smith
}

\begin{abstract}
Objective-To assess the risk of ischaemic stroke associated with total serum homocyst(e)ine (tHcy) concentration.

Design-Cohort study.

Setting-Caerphilly, South Wales

Participants-2254 men age 50 to 64 years recruited between 1984 and 1988 .

Results-107 men developed ischaemic stroke and mean follow up time was $\mathbf{1 0 . 2}$ years. There was no significant difference in mean serum total homocyst(e)ine levels between stroke cases $(12.2 \mu \mathrm{mol} 95 \%$ CI 11.6 to 13.1$)$ and non-cases $(11.7 \mu \mathrm{mol} 95 \%$ CI 11.5 to 11.9$)(p=0.14)$. There was no significant risk for a standard deviation increase in homocyst(e)ine (adjusted hazard ratio $=1.1,95 \%$ CI 0.9 to 1.4$)$. An interaction was observed between homocyst(e)ine and age at entry $(p=0.003)$. The adjusted odds ratio comparing the top quintile of homocyst(e)ine with the rest was $2.5(95 \%$ CI 1.0 to 6.2$)$ for strokes occurring under 65 years and $0.5(95 \% \mathrm{CI}$ 0.2 to 1.3 ) at 65 years or older ( $p$ value for interaction $=0.02$ ). Risk also differed by blood pressure status. The adjusted hazard ratio for a standard deviation increase in homocyst(e)ine was $0.8,(95 \%$ CI 0.6 to 1.2) for normotensive men and $1.3(95 \%$ CI 1.1 to 1.7 ) for hypertensive men (p value for interaction $=0.01$ ).

Conclusions-Overall, there is no significant relation between homocyst(e)ine and ischaemic stroke in this cohort. However, its aetiological importance may be greater for premature ischaemic strokes $(<65$ years) and in hypertensive men.

(F Epidemiol Community Health 2001;55:91-96)
\end{abstract}

Increased circulating total homocyst(e)ine (tHcy) concentration in the normal population occurs as a result of either minor genetic abnormalities or nutritional deficiencies of $\mathrm{B}$ vitamins such as folic acid. Recently, several studies have been published on the relation between increased serum tHcy concentration and atherosclerotic vascular disease and claims have been made that it is a strong independent risk factor. ${ }^{1}$ This observation is important because it is biologically plausible ${ }^{2}$ and because hyperhomocysteinaemia can be easily and cheaply treated with folic acid supplements. ${ }^{34}$ Despite the relative novelty of this risk factor, the "homocyst(e)ine hypothesis" has gathered such momentum that randomised controlled trials of folic acid are now being planned. ${ }^{5}$

Much of the positive evidence showing an association between serum tHcy concentration and atherosclerosis comes from studies of coronary heart disease (CHD) and is based on cross sectional ${ }^{6}$ and case-control studies. ${ }^{78}$ The evidence from cohort studies has been equivocal. Of 11 cohort studies, ${ }^{9-19} \operatorname{six}^{9-11} 141817$ found a positive significant association between increased serum tHcy concentration and CHD, and this disappeared in one of the studies with longer follow up time..$^{92}$ However, there is much less evidence examining the relation between increased serum tHcy concentration and ischaemic stroke. Cross sectional and case-control studies have reported positive findings ${ }^{82021}$ as have studies that measured carotid artery wall thickness as the primary outcome. ${ }^{22-24}$ There have been few prospective studies of the association between increased serum tHcy concentration and ischaemic stroke. The British Regional Heart Study ${ }^{25}$ in a cohort study of all stroke types showed a graded increase per quartile of serum tHcy concentration distribution with a significant trend. Recently the Framingham study has shown similar results with a significant trend across quartiles. ${ }^{26}$ The Rotterdam study of the elderly found a significant but modest association per $1 \mathrm{mmol}$ change in homocyst(e)ine (odds ratio 1.07, 95\% confidence intervals 1.03 to 1.12$).{ }^{17}$ The US Physicians Study failed to show a significant positive association ${ }^{27}$ as did a Finnish study. ${ }^{13}$ Recent systematic reviews have cautioned that there is too much heterogeneity between studies to calculate a quantitative summary estimate in a formal meta-analysis. This is mainly because of the study of diverse populations, little consistency in choice of confounders and use of data derived cut offs. ${ }^{28} 29$

\section{Methods}

DESIGN

The Caerphilly study is a community based prospective study of cardiovascular disease and related outcomes in men age 45 to 59 years who were recruited between 1979 and 1983 from the town of Caerphilly South Wales and the adjacent villages. ${ }^{30}$ All men in the eligible age group were identified from the electoral register (2818) and invited to participate with an $89 \%$ recruitment rate. In 1984, 2398 men aged 50 to 64 years participated in phase II of the study. Serum homocyst(e)ine concentration levels were performed on phase II stored blood samples at a later date. One hundred and forty four men were omitted from the analysis because of prevalent stroke (40), or unavailable serum tHcy concentration level (109) (5 were both). This analysis is based on 2254 men with no prior history of stroke. 
MEASUREMENT OF EXPOSURES

The phase II follow up collected the following measurements; medical history, smoking history, London School of Hygiene and Tropical Medicine chest pain questionnaire, height, weight and blood pressure measured with a random zero sphygmomanometer and a 12 lead electrocardiogram (ECG). Detailed methods for these and the wide range of other measures that were made are described elsewhere. ${ }^{30-32}$ For the purposes of this study social class was divided into manual and non-manual. Hypertension was defined as systolic blood pressure over $160 \mathrm{~mm} \mathrm{Hg}$, diastolic blood pressure $95 \mathrm{~mm} \mathrm{Hg}$ and/or self reported hypertension from the questionnaire.

A detailed food frequency questionnaire was self completed before attending the clinic and used to estimate the mean daily dietary intake of nutrients and vitamins. This was validated in a $30 \%$ sub-sample with a seven day weighted dietary intake. ${ }^{31}$ Participants were asked to return fasting within the next two weeks when a blood sample was obtained. Eighty eight men $(3.9 \%)$ were not fasting when the blood was taken.

LABORATORY METHODS

Serum was separated within two hours of obtaining the sample and stored at below $-20^{\circ}$ for between 6 and 10 years. Estimations of a wide range of factors relevant to cardiovascular disease were made and described elsewhere. ${ }^{30-32}$ Later, the blood samples were transported on dry ice to South Africa for serum tHcy concentration analysis. Serum total homocyst(e)ine concentration was obtained using a modified method by Araki and $S a^{33}$ of high performance liquid chromatography and was performed in duplicate on every sample. The mean of both measures was used in the analysis. One hundred and twelve samples were split and placed blindly throughout the sample series. The coefficient of variation for these duplicate samples was $9 \%$ when one outlier was omitted..$^{19}$

\section{STROKE/OUTCOME ASCERTAINMENT}

Deaths from stroke comprised all those coded as ICD 430-8 (9th revision of the International Classification of Diseases). Non-fatal strokes were defined clinically according to World Health Organisation criteria as all those cerebrovascular events that produced a neurological deficit that was present for more than 24 hours. Stroke type was then classified by scrutiny of all medical records, including neuroradiology, and where records were poor, the participant or a relative was visited to obtain more information. Summaries of symptoms, signs, past medical history and clinical course were reviewed independently by two clinicians (a stroke specialist and a clinical epidemiologist) and in cases of diagnostic disagreement a third stroke epidemiologist arbitrated. Definite strokes were classified as those that met full WHO criteria and these were further subdivided on the basis of neuroradiology or necropsy findings into infarction or haemorrhage. Probable strokes were those that did not meet full
WHO criteria but were diagnosed as strokes, and if symptoms or signs suggestive of haemorrhage were not present, they were classified as probable ischaemic strokes. Possible strokes were those in which confirmatory information was lacking but in which it was most probable that the underlying disorder was ischaemic. No attempt was made to distinguish lacunar or other sub-types of stroke from other ischaemic strokes as this is impracticable in epidemiological studies of this nature. Definite haemorrhagic strokes were excluded from the analyses but all other cases were included.

\section{STATISTICAL ANALYSIS}

Risk factors for stroke in cases and non-cases were examined using the unpaired $t$ test and $\mathrm{z}$ test in the comparison of means and proportions. Total homocyst(e)ine, vitamin B12 and alcohol were not normally distributed and all calculations were done on log transformed data.

Associations between serum tHcy concentration and several potential confounding variables were examined using linear regression. Mean values of serum tHcy concentration were compared in cases and non-cases using the unpaired $t$ test. To compare this analysis with other studies the relation between ischaemic stroke and serum tHcy concentration was examined using several approaches; (a) per unit/standard deviation increase to look for linear effects, ${ }^{13}{ }^{17}$ (b) trend across quintiles of the tHcy concentration distribution, ${ }^{25} 27$ (c) comparing the top quintile to the remaining $80 \%$ and top $5 \%$ to the bottom $95 \%$ to look for a threshold effect. ${ }^{27}$

Cox proportional hazard modelling was used to perform survival analysis and to adjust for confounders. Date of entry was taken as the date the blood was taken for serum total homocyst(e)ine. Participants were censored at date of ischaemic stroke, date of death or date of end of follow up, which was 31 December 1997.

The risk set was defined using both the dates of entry and age at entry and there was no difference in the results when analysed separately. The proportional hazard assumption for the goodness of fit of the Cox regression model was tested over three intervals of time each containing the same number of stroke events. This was tested for all explanatory variables both graphically using Aalen plots and statistically using the proportional hazard test.

In the final model adjustment is first made for strict confounders, which are age, diabetes, hypertension, systolic blood pressure and current smoking status. The model is then adjusted for factors strongly associated with stroke but not serum tHcy concentration, which are social class and ECG ischaemia. Finally, adjustment is made for factors associated with serum tHcy concentration such as folate, B12, B6, alcohol, body mass index and creatinine.

Interactions between serum tHcy concentration and other variables were explored in the regression model using the likelihood ratio test. Sub-group analyses were performed to assess 
Table 1 Characteristics of study population and risk factors for stroke

\begin{tabular}{|c|c|c|c|c|}
\hline \multicolumn{2}{|c|}{ Percentages } & $\begin{array}{l}\text { Cases } \\
(n=107) \%\end{array}$ & $\begin{array}{l}\text { Non-cases } \\
(n=2147) \%\end{array}$ & $p$ Value $Z$ test \\
\hline \multicolumn{2}{|c|}{ Social class, manual } & 76.4 & 66.9 & 0.04 \\
\hline \multirow[t]{6}{*}{ Smoking: } & ever & 90.6 & 81.4 & 0.01 \\
\hline & ex & 38.4 & 38.3 & 0.97 \\
\hline & current & 52.2 & 43 & 0.06 \\
\hline & $1-14$ cigarettes/day & 25.2 & 15.5 & $<0.01$ \\
\hline & 15-24 cigarettes/day & 14.0 & 12.0 & 0.52 \\
\hline & $>25$ cigarettes/day & 4.7 & 6.3 & 0.50 \\
\hline \multicolumn{2}{|c|}{ Hypertension } & 35.5 & 22.8 & $<0.01$ \\
\hline \multicolumn{2}{|c|}{ Diabetes (self report) } & 8.4 & 2.9 & $<0.01$ \\
\hline \multicolumn{2}{|c|}{ ECG ischaemia } & 32.7 & 14.6 & $<0.01$ \\
\hline \multicolumn{2}{|c|}{ Means } & & & $t$ test \\
\hline \multicolumn{2}{|c|}{ Age at entry (y) } & 59.2 & 57.4 & $<0.01$ \\
\hline \multicolumn{2}{|c|}{ Systolic blood pressure $(\mathrm{mm} \mathrm{Hg})$} & 154.2 & 146.1 & $<0.01$ \\
\hline \multicolumn{2}{|c|}{ Diastolic blood pressure (mm Hg) } & 86.9 & 84.8 & 0.08 \\
\hline \multicolumn{2}{|c|}{ Body mass index $\left(\mathrm{kg} / \mathrm{m}^{2}\right)$} & 26 & 25.9 & 0.84 \\
\hline \multicolumn{2}{|c|}{ Daily folate intake ( $\mu \mathrm{g} /$ day $)$} & 279.9 & 280.3 & 0.96 \\
\hline \multicolumn{2}{|c|}{ Daily B12 intake $(\mu \mathrm{g} / \text { day })^{\star}$} & 7.4 & 6.7 & 0.12 \\
\hline \multicolumn{2}{|c|}{ Daily B6 intake (mg/day) } & 1.2 & 1.2 & 0.42 \\
\hline \multicolumn{2}{|c|}{ Alcohol (ml/day) ${ }^{\star}$} & 59.7 & 57.5 & 0.80 \\
\hline \multicolumn{2}{|c|}{ Creatinine $(\mu \mathrm{mol} / \mathrm{l})$} & 100 & 99.2 & 0.44 \\
\hline
\end{tabular}

${ }^{\star}$ Geometric mean.

the association between stroke and serum tHcy concentration in young men as previous studies have suggested an interaction. ${ }^{17} 2527$ Because we wanted to test whether the relation between serum tHcy concentration differed for early ( $<65$ years) and later ( $\geqslant 65$ years) stroke, we used a nested case-control study design, so that cases could be defined by age at stroke, in contrast with age at entry for the proportional hazard model. Four controls per case were randomly selected from the comparison group if they were at risk of ischaemic stroke at the same age as the stroke case and using age frequency matching within one year. Logistic regression was used to determine the effect of serum tHcy concentration on stroke in different age strata adjusting for confounders. Interaction was tested using the likelihood ratio test. Subgroup analysis of those with and without hypertension was done using Cox proportional hazard modelling.

Table 2 Serum total homocyst(e) ine and potential confounding variables for ischaemic stroke in the non-cases

\begin{tabular}{|c|c|c|c|}
\hline Linear regression & $\beta$ coefficient $^{*}$ & $95 \% C I$ & $p$ Value \\
\hline Age (per 5 years) & 0.04 & 0.021 to 0.05 & $<0.01$ \\
\hline Systolic BP (per $10 \mathrm{~mm} \mathrm{Hg}$ ) & 0.006 & 0.006 to 0.012 & 0.03 \\
\hline Diastolic BP (per $10 \mathrm{~mm} \mathrm{Hg}$ ) & -0.0002 & -0.01 to 0.01 & 0.97 \\
\hline †Alcohol (per SD in ml/day) & -0.04 & -0.05 to -0.03 & $<0.01$ \\
\hline Body mass index (per SD in $\mathrm{kg} / \mathrm{m}^{2}$ ) & -0.1 & -0.14 to -0.07 & $<0.01$ \\
\hline Creatinine (per SD in $\mu \mathrm{mol} / \mathrm{l}$ ) & 0.06 & 0.04 to 0.07 & $<0.01$ \\
\hline Total cholesterol (per SD in $\mathrm{mmol} / \mathrm{l}$ ) & -0.0006 & -0.01 to 0.01 & 0.93 \\
\hline HDL cholesterol (per SD in $\mathrm{mmol} / \mathrm{l}$ ) & -0.02 & -0.03 to -0.007 & $<0.01$ \\
\hline †Triglycerides (per SD in mmol/1) & -0.01 & -0.02 to 0.02 & 0.1 \\
\hline Folate (per SD in $\mu \mathrm{g} /$ day) & -0.05 & -0.06 to -0.04 & $<0.01$ \\
\hline †B12 (per SD in $\mu \mathrm{g} /$ day) & -0.04 & -0.05 to -0.03 & $<0.01$ \\
\hline $\mathrm{B} 6$ (per SD in $\mathrm{mg}$ /day) & $\begin{array}{l}-0.02 \\
\neq M e a n \text { serum } t H c y \\
(\mu m / l)\end{array}$ & $\begin{array}{l}-0.04 \text { to }-0.01 \\
S D\end{array}$ & $<0.01$ \\
\hline Never smoked & 11.3 & 1.3 & \\
\hline Ex smoker & 11.5 & 1.3 & \\
\hline \multirow[t]{2}{*}{ Current smoker } & 12.1 & 1.4 & $\mathrm{p}=<0.01$ \\
\hline & $\begin{array}{l}\neq \text { \$Mean serum } t H c y \\
(\mu \mathrm{m} / \mathrm{l})\end{array}$ & $t$ test & \\
\hline Hypertensive & $\begin{array}{ll}12.1 & 1.3\end{array}$ & & \\
\hline Normotensive & 11.6 & $\mathrm{p}=0.01$ & \\
\hline Manual social class & 11.8 & & \\
\hline Non-manual social class & 11.5 & $\mathrm{p}=0.09$ & \\
\hline Diabetic & 10.7 & & \\
\hline Not diabetic & 11.7 & $\mathrm{p}=0.01$ & \\
\hline ECG ischaemia & 11.8 & & \\
\hline Normal ECG & 11.7 & $\mathrm{p}=0.5$ & \\
\hline
\end{tabular}

${ }^{\star}$ Change in homocysteine (natural log) per unit change in risk factor. $†$ Natural log transformed. $\ddagger$ Geometric mean.
Results

A total of 107 men had an ischaemic stroke and 2147 formed the comparison group. Forty four per cent (47) of ischaemic strokes were based on pathological and radiological evidence and $56 \%$ (60) based on clinical diagnosis. The mean follow up time was 10.2 years (range 3 days to 13.5 years) and 18 men $(16.4 \%)$ died from their first stroke within 30 days. The mean age at time of stroke was 65.2 years (SD $=5.2$ ).

Table 1 shows the risk factors for stroke in the cases and non-cases. Cases were more likely to be older, have hypertension, have a higher mean systolic blood pressure, be diabetic, have evidence of ECG ischaemia, be ever or current smokers and be of manual social class.

Table 2 shows the relation between serum tHcy concentration and potential confounding variables in the non-cases. The correlation coefficients correspond to the change in log transformed tHcy concentration with every unit change in risk factor. Established risk factors for ischaemic stroke such as smoking, hypertension, systolic blood pressure and age were positively associated with serum tHcy concentration. Alcohol, folate, vitamin B12, vitamin B6 and body mass index were inversely associated with serum tHcy concentration as was diabetes. There is a significant inverse relation between HDL cholesterol and tHcy concentration but no relation with total cholesterol or triglycerides. Mean serum tHcy concentration was slightly higher in men of manual social class but this was not statistically significant (table 2).

The geometric mean serum tHcy concentration was $12.24 \mu \mathrm{mol}$ in cases (95\% CI 11.6 to 13.1 ) and $11.7 \mu \mathrm{mol}$ in non-cases (95\% CI 11.5 to 11.9 ). The difference was not statistically significant $(p=0.14)$. In the Cox proportional hazard model, there was no evidence of departure from the assumption of proportionality. The adjusted hazard ratio of stroke per standard deviation change in serum tHcy concentration was $1.1(95 \%$ CI 0.9 to 1.4$) \mathrm{p}=0.16$. Table 3 illustrates the rate and adjusted rate ratio of stroke comparing each quintile of serum tHcy concentration with the first. There was no significant trend in rate ratio across quintiles of serum tHcy concentration. When the highest quintile of the serum tHcy concentration distribution was compared with the other four quintiles the adjusted hazard ratio for stroke was 1.3 (95\% CI 0.8 to 2.0 ) $\mathrm{p}=0.3$. The adjusted hazard ratio for stroke when the top $5 \%$ of the serum tHcy concentration distribution was compared with the rest was 2.0 (95\% CI 0.9 to 4.2 ) $\mathrm{p}=0.07$.

There was a significant interaction between serum tHcy concentration and age in the fully adjusted Cox proportional hazard model $(\mathrm{p}=0.003)$ when both were treated as continuous variables. The adjusted odds ratio of stroke when comparing the top quintile of the serum tHcy concentration distribution with the rest, stratified by age at stroke, was 2.5 (95\% CI 1.0 to 6.2$) \mathrm{p}=0.02$ in men who were under 65 years of age, as compared with 0.5 (95\% CI 0.2 to 1.2$) \mathrm{p}=0.16$ for men aged 65 years or over. 
Table 3 Hazard ratio of stroke comparing each quintile of serum total homocyst(e) ine concentration with the first

\begin{tabular}{lllllll}
\hline $\begin{array}{l}\text { Quintile of } \\
t H c y\end{array}$ & $n$ & Cases & $\begin{array}{l}\text { Mean } \\
t H c y\end{array}$ & $\begin{array}{l}\text { Rate } / 1000 \\
\text { person years }\end{array}$ & $\begin{array}{l}\text { Age adjusted } \\
\text { hazard ratio } \\
\text { (95\% CI) } \\
\text { (n=2112) }\end{array}$ & $\begin{array}{l}\text { Hazard ratio } \\
\text { adjusted }(95 \% \\
\text { CI })(n=2112)\end{array}$ \\
\hline 1 & 455 & 21 & 8.2 & 4.4 & 1.0 & 1.0 \\
2 & 454 & 21 & 10.0 & 4.5 & $1.2(0.6$ to 2.2$)$ & $1.3(0.7$ to 2.4) \\
3 & 444 & 23 & 11.4 & 5.0 & $1.3(0.7$ to 2.4$)$ & $1.3(0.7$ to 2.4) \\
4 & 453 & 14 & 13.2 & 3.0 & $0.8(0.4$ to 1.6$)$ & $0.8(0.4$ to 1.6$)$ \\
5 & 448 & 28 & 19.0 & 6.4 & $1.4(0.7$ to 2.6$)$ & $1.3(0.7$ to 2.6$)$ \\
$\mathrm{p}$ (trend) & & & & & 0.7 & 0.8 \\
\hline
\end{tabular}

*Adjusted for age, social class, current smoking, hypertension, systolic blood pressure, diabetes, ECG ischaemia, body mass index, creatinine, folate, vitimin B12, vitamin B6 and alcohol.

The interaction was statistically significant $(\mathrm{p}=0.02)$.

There was an interaction between serum tHcy concentration and diastolic blood pressure $(p=0.001)$. The adjusted hazard ratio of ischaemic stroke per standard deviation change in serum tHcy concentration for normotensive men was 0.8 (95\% CI 0.6 to 1.2$) \mathrm{p}=0.3$ versus $1.3(95 \%$ CI 1.0 to 1.7$) \mathrm{p}=0.002$ in hypertensive men. The interaction was again significant $(\mathrm{p}=0.01)$.

\section{Discussion}

In this study, a significant association between serum tHcy concentration and ischaemic stroke was not found although weak effect sizes cannot be excluded. The results were consistent when the top $5 \%$ and top $20 \%$ of the serum tHcy concentration distribution were used as cut off points and when tested for trend per quintile and per standard deviation change in serum tHcy concentration.

The Caerphilly study offers advantages over other studies of the relation between serum tHcy concentration and ischaemic stroke. Survival analysis is used and all data are fully exploited; unlike nested case-control studies all non-cases are part of the comparison group. Ascertainment of stroke status was very detailed and thorough and we have tried to only include ischaemic strokes as cases. We have assumed that those strokes of uncertain type were probably ischaemic. This assumption is reasonable given the age distribution of our events, so that ischaemic strokes would be the predominant type in this population. Initial misdiagnosis of stroke is quite common in clinical settings but is markedly improved by experience, neuroradiology and knowledge of the clinical course. ${ }^{34}$ Use of multiple clinical methods is considered sufficiently accurate for classifying haemorragic and ischaemic stroke for epidemiological purposes. ${ }^{35}$ In addition there was detailed measurement of potential confounders including renal function and dietary factors.

Out of five cohort studies that have been published, three have found a positive association between increased serum tHcy concentration and ischaemic stroke, the British Regional Heart Study, ${ }^{25}$ the Rotterdam study of the elderly ${ }^{17}$ and recently the Framingham study. ${ }^{26}$ None of these studies had dietary measures and none of them included only ischaemic strokes. Our results are consistent with the US Physician's study ${ }^{27}$ where the size
KEY POINTS

- Homocyst(e)ine has been gaining acceptance in the literature as a strong independent risk factor for coronary heart disease and stroke.

- The hypothesis has profound public health implications because homocyst(e)ine can be lowered cheaply and easily by folic acid supplements.

- Evidence from prospective cohort studies is conflicting.

- This cohort study does not support the hypothesis that homocyst(e)ine is associated with ischaemic stroke in men from the UK.

- Homocyst(e)ine may have some aetiological importance in premature strokes and in those with hypertension.

and the direction of the effect are very similar but the null hypothesis cannot be rejected because the confidence intervals include one. In contrast with the US Physician's study, which could be considered a selective well nourished study population, the Caerphilly cohort is composed of predominantly working class men.

If increased serum tHcy concentration is an independent risk factor for cerebrovascular disease as reported in other studies, then why did we not find an effect? Misclassification of exposure because of intraindividual variation of serum tHcy concentration is likely to be random and may have weakened the effect towards the null. A small non-fasting subgroup $(3.9 \%)$ and possible delay in separating serum may have contributed to this. Instability of stored assays is another possibility but homocyst(e)ine is now thought to be highly stable while frozen. ${ }^{36}$ Misclassification of outcome is another possibility as strokes of uncertain type were classified as ischaemic. However, the association between conventional risk factors and ischaemic stroke was very similar when strokes of uncertain type were included and excluded in separate analysis (data to be published elsewhere). Finally, our null results might be attributable to inadequate power as most previous studies show that any association is of very modest effect.

We observed a relatively high incidence of stroke in our study population. Age standardised mortality rates of stroke in England and Wales are higher than in the $\mathrm{USA}^{37}$ (MONICA). Age specific stroke death rates for age 45 to 65 years are higher in South Wales than in the England and Wales combined and lowest in the USA $(45,38.9,32.7$ per 100000 respectively). ${ }^{38-40}$ If the relation between tHcy and stroke is a strong independent causal one as suggested in the literature, then this population based study of predominantly working class men, with a high stroke incidence and reliable measurement of a large number of potential confounding factors is ideally suited to testing this hypothesis.

We performed sub-group analysis for two reasons. Firstly, other papers have reported 
extensively on a differential effect of age and hypertension on serum tHcy concentration. ${ }^{172527}$ However, more importantly we found a statistically significant interaction between serum tHcy concentration and age and between serum tHcy concentration and diastolic blood pressure. Sixty five years of age was chosen as a cut off because this gave similar numbers of strokes in both groups ( 50 cases under 65 years and 57 case above). We observed a stronger association between plasma tHcy and rate of ischaemic stroke in men who were under the age of 65 at the time of their stroke in comparison with those who were aged 65 years or over. One of the largest case-control studies of tHcy concentration and stroke was a study of 211 men with premature cerebrovascular disease in young men (mean age 43.7 years). They estimated an odds ratio of stroke of 1.7 (95\% CI 1.1 to 2.7) comparing the top quintile of the tHcy concentration distribution with the rest. ${ }^{8}$

It is possible that this interaction with age is attributable to chance, though it could well be a real effect. One possible explanation is that tHcy concentration as a risk factor in young men has fewer other risk factors to compete with. As people age, a greater number of risk factors contribute to the overall risk of disease and the apparent risk attributed to tHcy becomes weaker or negligible.

A far greater number of studies have examined the relation between $\mathrm{CHD}$ and tHcy. They are generally consistent in suggesting the possibility that there may be a differential age effect of tHcy. Almost all cross sectional and case-control studies of $\mathrm{CHD}$ with a positive finding have been of premature CHD with cases $<60$ years of age. ${ }^{741}$ In two cohort studies, which have found significant associations between tHcy and CHD, the mean age of cases was 53 years ${ }^{14}$ and 58 years. ${ }^{18}$ The British Regional Heart study is also a cohort of middle aged men. ${ }^{10}$

We found a greater risk of ischaemic stroke associated with tHcy in hypertensive men. The British Regional Heart Study ${ }^{25}$ and the Rotterdam study of the elderly found similar results but only the Rotterdam study found a significant interaction. In contrast, the US Physicians reported a greater risk in normotensive men than in those with hypertension ${ }^{27}$ as did another study of the elderly. ${ }^{42}$

Total homocyst(e)ine concentration is gaining wide acceptance in the literature as a strong independent risk factor for arterial occlusive disease, including stroke. It is important to recognise that not all cohort studies support this conclusion. A recent systematic review has pointed out that prospective cohort studies are less likely to find a positive result than case-control studies, particularly if they exclude pre-existing vascular disease. ${ }^{28}$ Our study supports the hypothesis that any true effect is weak or non-existent and may be only important in certain subgroups.

Future randomised controlled trials of folic acid supplementation may provide more robust evidence as to whether interventions that lower tHcy can prevent atherosclerosis and future risk of stroke. In view of our results, such trials would either require very large numbers of participants or recruit subjects at high risk.

Total homocyst(e)ine refers to the sum of the concentrations of free homocysteine, protein-bound homocysteine, the disulfide homocystine and the mixed disulfide homocysteine-cysteine. Una Fallon is a Wellcome Trust Training Fellow in Clinical Epidemiology.

Conflicts of interest: none.

1 Refsum H, Ueland PM, Nygard O, et al. Homocysteine and cardiovascular disease. Ann Rev Med 1998;49:31-62.

2 McCully KS. Homocysteine and vascular disease. Nat Med 1996;2:386-9.

3 Malinow MR, Duell PB, Hess DL, et al. Reduction of plasma homocyst(e)ine levels by breakfast cereal fortified with folic acid in patients with coronary heart disease. $N$ Engl f Med 1998;338:1009-15.

4 Ward $\mathrm{M}$, McNulty $\mathrm{H}$, McPartlin J, et al. Plasma homocysteine, a risk factor for cardiovascular disease, is lowered
by physiological doses of folic acid. $Q \mathcal{F}$ Med 1997;90:51924 .

5 Clarke R, Collins R. Can dietary supplements with folic acid or vitamin B6 reduce cardiovascular risk? Design of clinical trials to test the homocysteine hypothesis of vascular disease. F Cardiovasc Risk 1998;5:249-55.

6 Nygard O, Vollset SE, Refsum H, et al. Total plasma homocysteine and cardiovascular risk profile. The Hordaland cysteine and cardiovascular risk profile. The Horc
Homocysteine Study. $\mathcal{F A M A} 1995 ; 274: 1526-33$.

7 Clarke R, Daly L, Robinson K, et al. Hyperhomocysteinemia: an independent risk factor for vascular disease. $N$ Engl f Med 1991;324:1149-55.

8 Graham IM, Daly LE, Refsum HM, et al. Plasma homocysteine as a risk factor for vascular disease. The European Concerted Action Project. FAMA 1997;277: 1775-81.

9 Stampfer MJ, Malinow MR, Willett WC, et al. A prospective study of plasma homocyst(e)ine and risk of myocardial infarction in US physicians. $\mathscr{F} A M A 1992 ; 268: 877-81$.

10 Whincup PH, Refsum H, Perry IJ, et al. Serum total homocysteine and coronary heart disease: prospective study in middle aged men. Heart 1999;82:448-54.

11 Bostom AG, Silbershatz H, Rosenberg IH, et al. Nonfasting plasma total homocysteine levels and all-cause and cardiovascular disease mortality in elderly Framingham men and vascular disease mortality in elderly Framing

12 Chasan-Taber L, Selhub J, Rosenberg IH, et al. A prospecChasan-Taber $\mathrm{L}$, Selhub J, Rosenberg $\mathrm{IH}$, et al. A prospec-
tive study of folate and vitamin B6 and risk of myocardial infarction in US physicians. F Am Coll Nutr 1996;15:13643

13 Alfthan G, Pekkanen J, Jauhiainen M, et al. Relation of serum homocysteine and lipoprotein(a) concentrations to atherosclerotic disease in a prospective Finnish population based study. Atherosclerosis 1994;106:9-19.

14 Arnesen E, Refsum H, Bonaa KH, et al. Serum total homocysteine and coronary heart disease. Int $\mathcal{f}$ Epidemiol 1995;24:704-9.

15 Folsom AR, Nieto FJ, McGovern PG, et al. Prospective study of coronary heart disease incidence in relation to fasting total homocysteine, related genetic polymorphisms, and B vitamins: the Atherosclerosis Risk in Communities (ARIC) study. Circulation 1998;98:204-10.

16 Evans RW, Shaten BJ, Hempel JD, et al. Homocyst(e)ine and risk of cardiovascular disease in the Multiple Risk Factor Intervention Trial. Arterioscler Thromb Vasc Biol tor Intervention

17 Bots ML, Launer LJ, Lindemans J, et al. Homocysteine and short term risk of myocardial infarction and stroke in the elderly - The Rotterdam study. Arch Intern Med 1999;159: $38-44$.

18 Wald NJ, Watt HC, Law MR, et al. Homocysteine and ischemic heart disease: results of a prospective study with implications regarding prevention. Arch Intern Med 1998; 158:862-7.

19 Ubbink JB, Fehily AM, Pickering J, et al. Homocysteine and ischaemic heart disease in the Caerphilly cohort. Atherosclerosis 1998;140:349-56.

20 Brattstrom L, Lindgren A, Israelsson B, et al. Hyperhomocysteinaemia in stroke: prevalence, cause, and relationships cysteinaemia in stroke: prevalence, cause, and relationships
to type of stroke and stroke risk factors. Eur $\mathcal{f}$ Clin Invest to type of stroke

21 Coull BM, Malinow MR, Beamer N, et al. Elevated plasma homocyst(e)ine concentration as a possible independent risk factor for stroke. Stroke 1990;21:572-6.

22 Malinow MR, Nieto FJ, Szklo M, et al. Carotid artery intimal-medial wall thickening and plasma homocyst(e)ine in asymptomatic adults. The Atherosclerosis Risk in Communities Study. Circulation 1993;87:1107-13.

23 Selhub J, Jacques PF, Bostom AG, et al. Relationship between plasma homocysteine, vitamin status and extracranial carotid-artery stenosis in the Framingham Study population. $\mathcal{F}$ Nutr 1996;126:1258-65S.

24 Voutilainen S, Alfthan G, Nyyssonen K, et al. Association between elevated plasma total homocysteine and increased common carotid artery wall thickness. Ann Med 1998;30: 300-6.

25 Perry IJ, Refsum H, Morris RW, et al. Prospective study of serum total homocysteine concentration and risk of stroke in middle-aged British men. Lancet 1995;346:1395-8. 
26 Bostom AG, Rosenberg IH, Silbershatz H, et al. Nonfasting plasma total homocysteine levels and stroke incidence in elderly persons: the Framingham Study. Ann Intern Med 1999;131:352-5.

27 Verhoef P, Hennekens CH, Malinow MR, et al. A prospective study of plasma homocyst(e)ine and risk of ischemic stroke. Stroke 1994;25:1924-30.

28 Christen WG, Ajani UA, Glynn RJ, et al. Blood levels of homocysteine and increased risks of cardiovascular disease: causal or casual? Arch Intern Med 2000;160:422-34.

29 Hankey GJ, Eikelboom JW. Homocysteine and vascular disease. Lancet 1999;354:407-13

30 Anonymous. Caerphilly and Speedwell collaborative heart disease studies. The Caerphilly and Speedwell Collaborative Group 7 Epidemiol Commun Health 1984:38:259-62.

31 Fehily AM, Yarnell JW, Sweetnam PM, et al. Diet and incident ischaemic heart disease: the Caerphilly Study. $\mathrm{Br} \mathcal{F}$ Nutr 1993;69:303-14.

32 Yarnell JW, Baker IA, Sweetnam PM, et al. Fibrinogen, viscosity, and white blood cell count are major risk factors for ischemic heart disease. The Caerphilly and Speedwell colischemic heart disease. The Caerphilly and Speedwell collaborati

33 Ubbink JB, Hayward VW, Bissbort S. Rapid highperformance liquid chromatographic assay for total homo- cysteine levels in human serum. 7 Chromatogr 1991;565: $441-6$

4 Norris JW, Hachinski VC. Misdiagnosis of stroke. Lancet 1982;i:328-31.

35 Ebrahim S, Harwood R. Stroke: epidemiology, evidence and clinical practice. Oxford: Oxford University Press, 1999:80. 36 Lindgren A, Brattstrom L, Norrving B, et al. Plasma homoysteine in the acute and convalescent phases after stroke. Stroke 1995;26:795-800.

37 Khaw KT. Epidemiology of stroke. I Neurol Neurosurg Psychiatry 1996;61:333-8.

38 Health Statistics Wales 1998. Wales: A publication of the Government Statistical Service, 1999

39 Office of National Statistics 1998 Mortality Statistics, Cause. England and Wales. London: A publication of the Government Statistical Service, 1999

40 FASTATS Stroke. http://www.cdc.gov/nchs/fastats/ stroke.htm. National Centre for Health Statistics, Centre for Disease Control and Prevention, 1999.

41 Israelsson B, Brattstrom LE, Hultberg BL. Homocysteine and myocardial infarction. Atherosclerosis 1988;71:227-33.

42 Stehouwer CD, Weijenberg MP, van den Berg M, et al. Serum homocysteine and risk of coronary heart disease follow-up. Arterioscler Thromb Vasc Biol 1998;18:1895-901. 